\title{
Postnatal infection is associated with widespread abnormalities of brain development in premature newborns
}

\author{
Vann Chau' ${ }^{1}$, Rollin Brant ${ }^{2}$, Kenneth J. Poskitt ${ }^{1,3}$, Emily W.Y. Tam ${ }^{4}$, Anne Synnes ${ }^{1}$ and Steven P. Miller ${ }^{1,4}$
}

INTRODUCTION: Infection is a risk factor for adverse neurodevelopmental outcome in preterm newborns. Our objective was to characterize the association of postnatal infection with adverse microstructural and metabolic brain development in premature newborns.

RESULTS: In 34/117 newborns studied, clinical signs were accompanied by positive cultures whereas 17 had clinical signs of sepsis alone. White matter injury (WMI) was identified in 34 newborns. In multivariate regression models, infected newborns had brain imaging measures indicative of delayed brain development: lower $\mathrm{N}$-acetylaspartate/choline, elevated average diffusivity $\left(D_{A V}\right)$, and decreased white matter fractional anisotropy. These widespread brain abnormalities were found in both newborns with positive-culture infection and in those with clinical infection.

DISCUSSION: These findings suggest that postnatal infection, even without a positive culture, is an important risk factor for widespread abnormalities in brain development. These abnormalities extend beyond brain injuries apparent with conventional magnetic resonance injury (MRI).

METHODS: 117 preterm newborns (24-32 wk gestation) were studied prospectively at a median of 32.0 and 40.3 wk ostmenstrual age with MRI (WMI, hemorrhage), magnetic resonance (MR) spectroscopy (metabolism), and diffusion tensor imaging (microstructure). Newborns were categorized as having "no infection," "clinical infection," or "positive-culture infection." We compared brain injuries as well as metabolic and microstructural development across these infection groups.

W hite matter injury (WMI) is the most common pattern of brain injury identified in the premature newborn $(1,2)$. Perinatal infection is now recognized as an important risk factor for WMI in this population (1-4). Of particular relevance to neonatal care, almost half of premature newborns with postnatal infection have neurodevelopmental impairments on follow-up, even when the infection is only evident clinically without positive cultures (5). It is unknown whether these "clinical" infections also increase the risk of brain injury in the premature newborn. Furthermore, although some studies suggest that the neurodevelopmental effects of postnatal infection are mediated by WMI (3), others do not (1). Recent evidence using diffusion tensor tractography in premature newborns suggests that postnatal infections are associated with abnormal development of the corticospinal tract as the newborns mature to "term-equivalent" age (6). It is thus imperative to determine whether postnatal infections, even in the absence of positive cultures, are associated with more widespread abnormalities in early brain development.

Advanced magnetic resonance (MR) techniques, such as spectroscopic imaging and diffusion tensor imaging, now enable serial in vivo assessments of brain metabolic and microstructural development in premature newborns (7). Using state-of-the-art brain imaging and detailed characterization of infection in a prospective cohort of premature newborns, we addressed the hypothesis that postnatal infection precedes early widespread abnormalities of brain development.

\section{RESULTS}

Postnatal Infection and Other Risk Factors for Brain Injury Fifty-one newborns (44\%) were infected before the first scan: 17 had clinical infection whereas 34 had positive-culture infection. Of those with positive-culture infection, 13 newborns had sepsis with necrotizing enterocolitis (NEC) and 4 had sepsis with meningitis. Some patients had multisystem infections or were infected with more than one organism. Of all "positiveculture infections" up to term age, most were sepsis (36 cases). Urinary tract infection, pneumonia, and meningitis were found in 9, 6, and 4 newborns, respectively. The most common pathogen was Staphylococcus species (30 cases), followed by $E$. coli (6), C. albicans (6), Enterococcus species (6), Klebsiella species (3), and others (4). Twenty-four newborns had multiple infections during their neonatal intensive care unit stay.

Compared with non-infected neonates, those with postnatal infection were younger and smaller at birth, with more systemic illness (Table 1). Among 44 newborns with hypotension, 11 experienced it during an infection episode, 5 during a NEC episode, and the remainder during the first week of life.

\section{Postnatal Infection and Brain Injury}

WMI was found in 34 (29\%) newborns: 10 mild, 14 moderate, and 10 severe. Cystic periventricular leukomalacia

\footnotetext{
'Department of Pediatrics, University of British Columbia, Vancouver, British Columbia, Canada; ${ }^{2}$ Department of Statistics, University of British Columbia, Vancouver, British Columbia, Canada; ${ }^{3}$ Department Radiology, University of British Columbia, Vancouver, British Columbia, Canada; ${ }^{4}$ Department of Neurology, University of California, San Francisco, San Francisco, California. Correspondence: Steven P. Miller (smiller6@cw.bc.ca)
} 
was found on clinical head ultrasound in only 4 newborns. On univariable analysis, the presence of postnatal infection prior to the first scan was associated with an increased risk of cerebellar hemorrhage (Table 1 ). In a multivariable model adjusting for gestational age (GA) at birth and birth weight, the risk of WMI on the first scan increased with positiveculture infection (odds ratio $(\mathrm{OR})=3.1 ; 95 \%$ confidence interval (CI): 1.04 to $9.4 ; P=0.04)$ but not with clinical infection alone $(\mathrm{OR}=2.1 ; 95 \% \mathrm{CI}$ : 0.6 to $7.7 ; P=0.3)$. We then examined the relationship of positive culture infections with WMI on the first scan adjusting for GA at birth, birth weight, patent ductus arteriosus, NEC, hypotension, and days of mechanical ventilation: positive-culture infections remained a significant risk for WMI (OR = 3.4; 95\% CI: 1.06 to $10.8 ; P=0.04)$. Of the four newborns with meningitis, two had recurrent episodes of sepsis, and all had WMI (one mild, two moderate, one severe). The risk of cerebellar hemorrhage increased with positive-culture infection $(\mathrm{OR}=8.9$; 95\% CI: 1.4 to $55.9 ; P=0.02)$ as well as with clinical infection alone $(\mathrm{OR}=15.7 ; 95 \% \mathrm{CI}: 1.8$ to $133.4 ; P=0.01)$ when adjusting for GA at birth, birth weight, and intraventricular hemorrhage severity.

\section{Postnatal Infection: Association With Abnormal Brain Development}

When examining brain metabolic development from the first to the term-equivalent MR scans with adjustment for age at scan, regions of interest, and presence of WMI, postnatal infection was found to be associated with significantly lower $\mathrm{N}$-acetylaspartate (NAA)/choline; this effect was especially pronounced on the second scan. Newborns with postnatal infection had 5.2\% lower NAA/choline on the first scan (95\% CI: -9.1 to $-1.2 \% ; P=0.01$ ) and $12.7 \%$ lower NAA/choline on the second (95\% CI: -16.5 to $-8.9 \% ; P<0.0001)$. In longitudinal models, newborns with postnatal infections had a slower rate of increase in NAA/choline than newborns without a postnatal infection: NAA/choline was a further $7.5 \%$ lower in newborns with infection on the second scan relative to the first (95\% CI: -11.2 to $-3.8 \%$; $P<0.0001)$. These effects were similar when newborns with meningitis were excluded from the model. The alterations in NAA/choline did not

Table 1. Clinical characteristics of the newborns with and without postnatal infection (prior to the first MR scan)

\begin{tabular}{|c|c|c|c|c|}
\hline Median (interquartile range) or number (\%) & No infection & Clinical infection & Positive-culture infection & $P$ value \\
\hline Number & 66 & 17 & 34 & \\
\hline \multicolumn{5}{|l|}{ Prenatal clinical features } \\
\hline Male & $30(45 \%)$ & $8(47 \%)$ & $19(56 \%)$ & 0.6 \\
\hline Gestational age at birth (wk) & $29.0(27.0-30.4)$ & $27.3(26.3-27.7)$ & $25.9(25.7-27.9)$ & 0.0001 \\
\hline Birth weight (g) & $1,155(925-1,380)$ & $945(805-1,120)$ & $844(731-955)$ & 0.0001 \\
\hline Head circumference $(\mathrm{cm})$ & $26.5(24.2-28.2)$ & $25.0(22.5-25.5)$ & $24(22.5-25.0)$ & 0.0002 \\
\hline Histopathological chorioamnionitis & $17(26 \%)$ & $10(59 \%)$ & $11(32 \%)$ & 0.05 \\
\hline \multicolumn{5}{|l|}{ Early postnatal clinical features } \\
\hline Cesarean delivery & $34(52 \%)$ & $14(82 \%)$ & $21(62 \%)$ & 0.06 \\
\hline Apgar score at 5 min & $8(6-9)$ & $7(7-8)$ & $7(6-8)$ & 0.4 \\
\hline Hyaline membrane disease & $47(71 \%)$ & $16(94 \%)$ & $33(97 \%)$ & 0.002 \\
\hline \multicolumn{5}{|l|}{ Late postnatal clinical features } \\
\hline Necrotizing enterocolitis & $5(8 \%)$ & $6(35 \%)$ & $13(38 \%)$ & $<0.001$ \\
\hline Patent ductus arteriosus & $21(32 \%)$ & $11(65 \%)$ & $24(71 \%)$ & $<0.001$ \\
\hline Hypotension & $15(23 \%)$ & $11(65 \%)$ & $18(53 \%)$ & 0.001 \\
\hline Intubation (days) & $1(0-9)$ & $11(3-36)$ & $35.5(6-62)$ & 0.0001 \\
\hline Chronic lung disease & $8(12 \%)$ & $8(47 \%)$ & $21(62 \%)$ & $<0.001$ \\
\hline \multicolumn{5}{|l|}{ Brain imaging } \\
\hline Postmenstrual age at 1 st scan (wk) & $31.9(30.3-33.0)$ & $30.6(29.6-33.6)$ & $32.4(30.9-34.6)$ & 0.04 \\
\hline White matter injury & $16(24 \%)$ & $5(29 \%)$ & $13(38 \%)$ & 0.3 \\
\hline Intraventricular hemorrhage (any grade) & $28(42 \%)$ & $9(53 \%)$ & $18(53 \%)$ & 0.4 \\
\hline Cerebellar hemorrhage & $2(3 \%)$ & $4(24 \%)$ & $8(24 \%)$ & 0.003 \\
\hline \multicolumn{5}{|l|}{ Neurological outcome at term-equivalent age } \\
\hline \multicolumn{5}{|l|}{ Clinical outcomes available in 109 newborns } \\
\hline Abnormal neuromotor score & $32(56 \%)$ & $13(93 \%)$ & $26(87 \%)$ & 0.001 \\
\hline G-tube feed & $11(18 \%)$ & $10(67 \%)$ & $23(72 \%)$ & $<0.001$ \\
\hline Respiratory support ${ }^{\mathrm{a}}$ & $1(2 \%)$ & $1(7 \%)$ & $5(16 \%)$ & 0.02 \\
\hline
\end{tabular}

MR, magnetic resonance.

aRespiratory support: oxygen therapy, continuous positive airway pressure/synchronized intermittent positive airway pressure, or mechanical ventilation. 
differ significantly in newborns with clinical infection relative to those with positive-culture infections $(P=0.59)$. Furthermore, the association of postnatal infections with lower NAA/choline did not differ meaningfully across brain regions. In an expanded model that included potential clinical confounding variables (GA at birth, birth weight, patent ductus arteriosus, NEC, hypotension, and days of mechanical ventilation), postnatal infections remained significant associated with lower NAA/choline: 4.6\% lower NAA/choline on the first scan $(P=0.019)$ and $7.0 \%$ lower NAA/choline on the second $(P=0.001)$.

Lactate/choline did not differ significantly in newborns with and without postnatal infection with adjustment for GA at scan and presence of WMI $(P=0.28)$.

When examining measures of white matter microstructural development from the first to the term-equivalent MR scan with adjustment for GA at scan and presence of WMI, fractional anisotropy (FA) was lower in newborns with infection relative to newborns without infection (overall: $-6.5 \%$; 95\% CI: -11.2 to $-1.8 \% ; P=0.006)$. There was a significant interaction of infection and white matter regions, with the most pronounced difference observed in the posterior white matter (Figure 1). The effect of infection on FA did not differ significantly between newborns with clinical infection alone and those with positive-culture infection $(P=0.63)$. Yet when the four newborns with meningitis were excluded from the model, the association of infection and FA was attenuated: FA was $4.5 \%$ lower in newborns with infection (95\% CI: -9.4 to $0.01 \%$; $P=0.06)$. In the expanded model that included potential clinical confounding variables, postnatal infections remained significantly associated with lower overall FA $(-8.4 \% ; P<0.0001)$. The reduced FA observed in infected newborns resulted from an increase in radial diffusivity (mean lamda2 \& lamda3) $\lambda_{2} / \lambda_{3}$ relative to axial diffusivity (lamda1) $\lambda_{1}$ in infected newborns.

In a similar model, brain average diffusivity $\left(\mathrm{D}_{\mathrm{AV}}\right)$ in white matter regions was $5.1 \%$ higher in the newborns with postnatal infection on the second scan (95\% CI: 3.2 to $7.0 \% ; P<0.0001)$. There was no significant interaction of infection with GA at scan on $\mathrm{D}_{\mathrm{AV}}$ nor did the association of postnatal infections with higher $\mathrm{D}_{\mathrm{AV}}$ differ meaningfully across white matter regions. Average diffusivity in gray matter regions on the second scan did not differ in newborns with postnatal infection $(P=0.44)$. The association of infection with $\mathrm{D}_{\mathrm{AV}}$ was similar when newborns with meningitis were excluded from the model. In the expanded model that included potential clinical confounding variables, postnatal infections remained significantly associated with higher white matter $\mathrm{D}_{\mathrm{AV}}$ on the second scan $(5.4 \%$; 95\% CI: 2.1 to $8.7 \% ; P=0.001$ ).

\section{Postnatal Infections and Early Neurological Outcomes}

All newborns in this cohort except one survived to termequivalent age with clinical outcomes available in 109 (93\%). As compared with noninfected newborns at term-equivalent age, the newborns who were exposed to postnatal infection were more likely to have chronic lung disease, have higher neuromotor scores (i.e., were neurologically more impaired), and need respiratory support and G-tube feeding (Table 1).

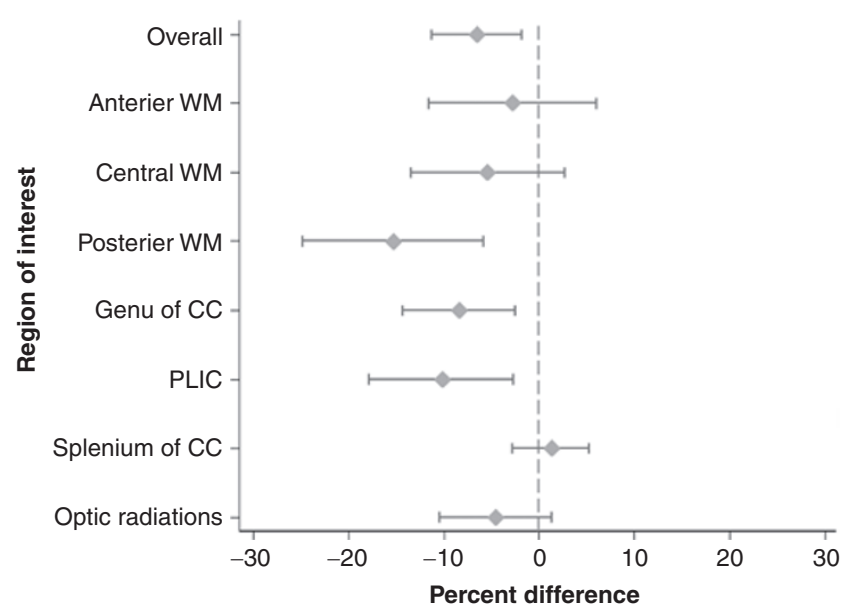

Figure 1. Fractional anisotropy: Infected newborns relative to noninfected newborns. The mean difference, with 95\% confidence intervals, is plotted for the overall effect and each region of interest in the white matter. CC, corpus callosum; PLIC, posterior limb of the internal capsule; WM, white matter.

\section{DISCUSSION}

The observation that infections affecting premature newborns in the neonatal intensive care unit precede widespread abnormalities of brain development, in addition to brain injury on magnetic resonance imaging (MRI) (e.g., WMI and cerebellar hemorrhage), has a number of important implications for the care of these newborns. Widespread abnormalities of brain development are seen in newborns with postnatal infection and are independent of extreme prematurity and common neonatal comorbidities. These brain abnormalities are seen even when the infection is only clinical without positive cultures. These findings are consistent with the significant increase in risk for adverse neurodevelopmental outcomes observed in premature newborns; deficits in motor and cognitive function are most apparent in premature newborns with postnatal infections, even when they are only evident clinically. Of note, the full spectrum of brain abnormalities related to infection was not apparent on conventional MRI nor was it fully apparent on the earliest MR evaluation. With serial quantitative brain imaging, the impact of postnatal infection was most readily detected at term-equivalent age, suggesting an impairment of ongoing brain development. Thus, rigorous neurodevelopmental follow-up of these high-risk newborns is indicated, even in the absence of positive bacteriologic cultures or WMI on MRI. These data also provide novel insights into how infection affects the brain and points to the critical need not only to prevent WMI but also to optimize brain development following a postnatal infection.

\section{Early Brain Development: The Effect of Infection}

The in vivo demonstration that postnatal infection is associated with widespread abnormalities of brain development that become more apparent over time builds on the current concept that infection and inflammation constitute one of the major initiating mechanisms in the pathogenesis of injury to the immature brain. Markers of intrauterine T-cell activation 
and increased proinflammatory cytokine concentrations in the serum and cerebrospinal fluid are seen in preterm newborns with WMI on MRI (8). Some of these cytokines appear to be toxic to the developmentally regulated cell populations prevalent in the immature white matter (9). Their presence may also induce a microglial response with generation of free radicals and glutamate-mediated excitoxicity (10). Experimental and clinical studies have demonstrated that "endotoxin," an exogenous polysaccharide, activates a cascade of immune reactions that are highly deleterious to the survival of pre-oligodendrocytes by binding and interacting with toll-like receptors $(11,12)$. The widespread abnormalities of brain metabolic and microstructural development detected in our study are a plausible consequence of this inflammatory insult. The widespread abnormalities in brain development observed in this cohort following infection are consistent with the changes in corticospinal tract development observed in a smaller subset of these newborns studied serially with diffusion tensor tractography (6). Together, these findings may explain the observation of "progressive" WMI following postnatal infection (4), given that the vulnerable cell population (the oligodendroglia progenitor) may have failed to mature to the "resistant" myelin-forming oligodendrocyte.

The association of postnatal infection with NAA/choline and $\mathrm{D}_{\mathrm{AV}}$ persisted even when the four newborns with meningitis were excluded. In contrast, the association of postnatal infection with white matter FA values was attenuated when the four newborns with cerebrospinal fluid-confirmed meningitis were excluded. This suggests that meningitis affects white matter microstructure in a manner beyond that visualized on conventional MRI as WMI.

The recent finding that chorioamnionitis (i.e., antenatal infection) was not a significant risk factor for abnormal brain development $(7,13)$ contrasts with the association of postnatal infection with impaired brain development. The germs involved in chorioamnionitis and postnatal infections may differ $(2,3)$, although placental culture was not always available in previous studies. Outside the intrauterine environment, the newborn may be more vulnerable to the consequences of infection. This may relate to an increased susceptibility to other mediating factors such as more intensive need for mechanical ventilation, limited nutrition, and hypotension (14).

The mechanism for white matter abnormalities in culturenegative, clinically infected infants remains poorly understood. In addition to inflammation, a second upstream mechanism in the pathogenesis of WMI is hypoxia-ischemia (10). In our cohort, hypotension was seen with similar frequency in newborns with clinical sepsis and in those with positive cultures. Growing evidence suggests that pressure-passive cerebral circulation is common in premature newborns (15). Cerebrovascular autoregulation in the developing brain seems vulnerable to exogenous factors, and the presence of systemic cytokines, triggered by an episode of sepsis or NEC, may lead to its disturbance (16). In experimental models, it is recognized that infection and inflammation potentiate the effects of hypoxia-ischemia (17). Thus, white matter abnormalities related to infection, with or without positive cultures, may relate to inflammation or disturbed cerebral blood flow, or some combination of the two.

Together, our findings stress the need to prevent and treat postnatal infection in premature newborns given its potential devastating impact on neurological outcomes (5). As suggested by a cluster randomized control trial, continuous quality-improvement methods can reduce nosocomial infection rates in the neonatal intensive care unit (3). Our data suggest that even clinical sepsis with negative cultures significantly affects brain development. This finding is in keeping with recent data showing that presumed and definite bacteremia in extremely low GA infants are associated with similar postnatal comorbidities and highlights the importance of this common condition (18). Recent data from experimental models suggest the potential for novel therapeutic approaches to prevent the white matter abnormalities associated with sepsis (19).

\section{Limitations}

The clinical characteristics of our cohort, including the rates of WMI $(1,20,21)$ and postnatal infection $(3,22)$, are similar to those of other groups and suggest that our findings are applicable to other preterm populations even though the study was carried out in a single center. Due to their medical condition, newborns with positive-culture infections were generally scanned at a later chronological age. Although all analyses were adjusted for postmenstrual age at scan, if there were residual confounding, the age difference would have nonetheless biased the newborns with infection to appear more mature, suggesting that differences might have been underestimated. The impact of these striking differences in the MRI outcomes between the newborns with and without postnatal infection on the character and severity of adverse long-term neurodevelopmental outcomes will be examined with long-term follow-up of this cohort.

\section{Conclusions}

Postnatal infection, even without a positive culture, is an important risk factor for widespread abnormalities in brain metabolic and microstructural brain development. These abnormalities extend beyond brain injuries apparent with conventional MRI, such as WMI. The finding that postnatal infection is an important risk factor for altered brain development has direct and critical clinical implications due to the treatable and preventable nature of this condition.

\section{METHODS}

\section{Study Population}

This study was approved by the University of British Columbia Clinical Research Ethics Board. Newborns were recruited prospectively, and informed consent was obtained from parents/guardians, at the British Columbia Women's Hospital, the major provincial tertiary-level neonatal referral center, from April 2006 to May 2009. Earlier stages of this cohort were described previously to address the relationship of chorioamnionitis with brain injury and to characterize corticospinal tract development $(6,7)$. Newborns were eligible if they were delivered between 24 and $32 \mathrm{wk}$ of gestation but were excluded if they had (i) clinical evidence of a congenital malformation or syndrome, 
(ii) antenatal congenital infections, or (iii) ultrasound evidence of a large parenchymal hemorrhagic infarction $(>2 \mathrm{~cm})(23)$. Of the parents of eligible newborns approached, 117 (54\%) consented to participate in this study. Enrolled patients were slightly younger (median: 27.0 vs. 28.9 wk GA; $P=0.003$ ) and smaller (median: 1,020 vs. $1,125 \mathrm{~g}$; $P=0.01)$ at birth, as compared with those not participating.

\section{MRI Studies}

A total of 117 newborns delivered at a median GA of $27.6 \mathrm{wk}$ (interquartile range: $26.0-29.6 \mathrm{wk}$ ) were scanned using an MR-compatible isolette (Lammers Medical Technology, Luebeck, Germany) and specialized neonatal head coil (Advanced Imaging Research, Cleveland, $\mathrm{OH})$. Newborns were scanned as soon as they were clinically stable and at median postmenstrual age of $32.0 \mathrm{wk}$ (interquartile range: $30.3-33.6 \mathrm{wk})$, and 97 were scanned again at term-equivalent age (median of $40.3 \mathrm{wk}$ (interquartile range: $38.7-42.7 \mathrm{wk}$ )). Detailed imaging methods applied in this cohort have been described previously (7). MRI studies were carried out without pharmacological sedation on a Siemens 1.5 Tesla Avanto scanner with 3D coronal volumetric $\mathrm{T}_{1}$-weighted images and axial fast-spin echo $\mathrm{T}_{2}$-weighted images (7). An experienced neuroradiologist, blinded to the newborn's medical history, reviewed the images. The severity of WMI, intraventricular hemorrhage, and cerebellar hemorrhage were

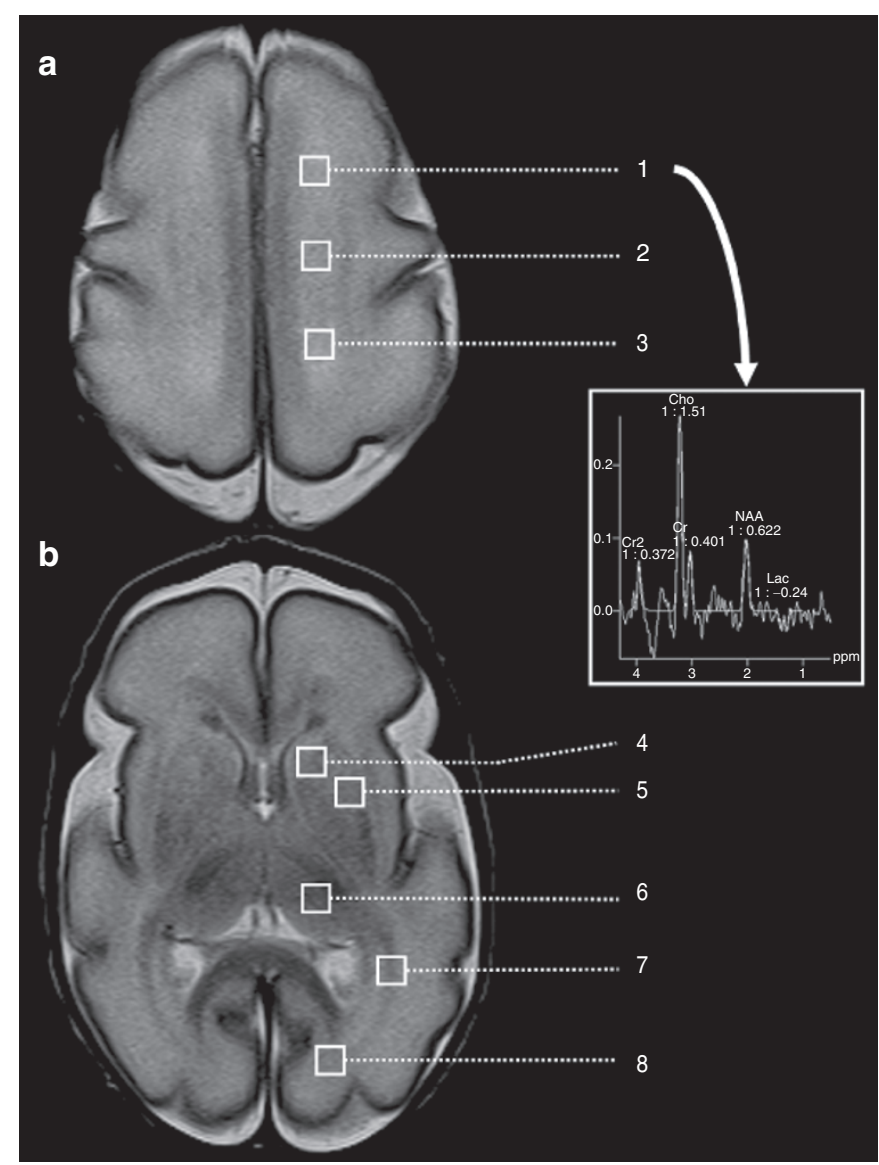

Figure 2. Proton magnetic resonance imaging and regions of interest. Brain metabolic development was assessed using magnetic resonance spectroscopic imaging in eight regions of interest at the level of (a) the high centrum semi-ovale and (b) the basal ganglia: high white matter ((1) anterior, (2) central, and (3) posterior), (4) caudate, (5) lentiform nuclei, (6) thalamus, (7) optic radiations, (8) calcarine region. The values of each region were averaged bilaterally. The arrow shows the spectrum of the left frontal white matter. This example is taken from a premature newborn with a normal magnetic resonance imaging born at 27-wk gestation and scanned at 28-wk postmenstrual age. Cho, choline; Cr, creatine; Lac, lactate; NAA, N-acetylaspartate. recorded as described previously (7). MR spectroscopic imaging was used to assess brain metabolism by measuring metabolite ratios in eight anatomical regions (Figure 2) (7). NAA/choline reflects neuronal integrity and metabolism, and increases with brain maturation (24). The lactate/choline reflects oxidative metabolism and is usually undetectable by term-equivalent age (24). As absolute metabolite quantification is not possible with our MR spectroscopic imaging technique, metabolite ratios were used to account for potential changes in intrinsic MR properties (in particular $\mathrm{T}_{2}$ ) of NAA and lactate with hypoxia-ischemia. Diffusion tensor imaging measures reflect microstructural brain development (25). As the brain matures, average diffusivity decreases due to developing neuronal and glial cell membranes that hinder water diffusion (26). With white matter development, FA increases with maturation of the oligodendrocyte lineage and early myelination (26). These parameters were measured from seven white matter and five gray matter regions (Figure 3) (7) (Supplementary Methods online).

\section{Postnatal Infection}

Postnatal infection (occurring $>3 \mathrm{~d}$ of life) was recorded using the categories proposed by Stoll et al.: (i) absence of infection, (ii) clinical infection alone, (iii) positive-culture infection, (iv) combination of NEC and positive-culture sepsis, and (v) meningitis with or without positive-culture sepsis (5). Culture was "positive" if a pathogen was found in the blood, urine, or cerebrospinal fluid. With clinical respiratory infection, $\geq 4$ white blood cells per field associated with a specific pathogen in the tracheal aspirates was considered to be a positive culture. Given the small number of newborns in this cohort, categories 3, 4, and 5 were grouped as "positive-culture infection." As not all newborns had routine lumbar punctures, we did not exclude newborns with meningitis; rather, we examined whether the final statistical models differed when newborns diagnosed with meningitis were removed. Other clinical data were collected systematically by chart review (7).

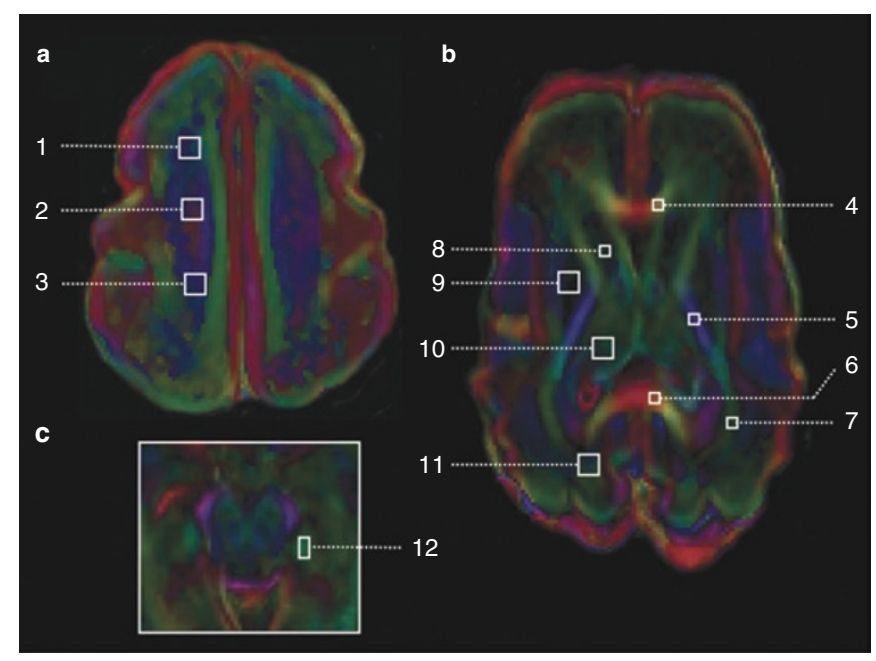

Figure 3. Diffusion tensor imaging and regions of interest. Brain microstructural development was assessed using diffusion tensor imaging in 12 regions of interest at the level of (a) the high centrum semi-ovale, (b) the basal ganglia, and (c) the midbrain, including high white matter ((1) anterior, (2) central, and (3) posterior), (4) genu of the corpus callosum, (5) posterior limb of the internal capsule, (6) splenium of the corpus callosum, (7) optic radiation, (8) caudate, (9) lentiform nuclei, (10) thalamus, (11) calcarine region, and (12) hippocampus. The values of each region were averaged bilaterally. This example is taken from a premature newborn with a normal magnetic resonance imaging born at 27-wk gestation and scanned at 28-wk postmenstrual age. In the color convention used to display the predominant diffusion direction, red represents right-left, green represents anterior-posterior, and blue represents superior-inferior anatomical directions. 


\section{Data Analysis}

We hypothesized that postnatal infection, even in the absence of positive culture, is associated with widespread abnormalities of early brain development. Statistical analysis was performed using Stata 9.2 (Stata, College Station, TX) and R version $2.11(27,28)$. Clinical characteristics of the newborns were compared using Fisher's exact test and the Kruskall-Wallis test for categorical and continuous data, respectively. The association of postnatal infection and other clinical variables with WMI was tested with logistic regression. The mean values of NAA/choline and lactate/choline ratios, and $\mathrm{D}_{\mathrm{AV}}$ and FA were compared among the newborns without infection, those with clinical infection, and those with positive-culture infection, in a generalized least squares regression model for repeated measures, adjusting for postmenstrual age at MRI scan, multiple regions of interest, and presence of WMI. A log-transformed outcome variable was used to determine the percentage differences of the MR measures. Interaction terms were examined (i) to determine whether postnatal infection modified the change of these MR parameters over time and (ii) to explore whether the effect of infection varied across regions. Interaction terms that improved predictive performance as assessed by the Akaike Information Criterion were incorporated into reported estimates (29). To explore the impact of potential clinical confounding variables on the association of postnatal infection with measures of brain development, we examined an expanded model that included terms for GA at birth, birth weight, patent ductus arteriosus, NEC, hypotension, and days of mechanical ventilation.

SUPPLEMENTARY MATERIAL is linked to the online version of the paper at http://www.nature.com/pr

\section{ACKNOWLEDGMENTS}

We thank the children and their parents who generously participated in this study.

\section{STATEMENT OF FINANCIAL SUPPORT}

This work is supported by a Canadian Institutes for Health Research (CIHR) operating grant (MOP 79262). S.P.M. is supported by a Canada Research Chair in Neonatal Neuroscience and by CIHR Clinician-Scientist and Michael Smith Foundation for Health Research Scholar awards. E.W.Y.T. is a Cerebral Palsy International Research Foundation Ethel \& Jack Hausman Clinical Research Scholar.

Disclosure: The authors have no conflict of interest or potential financial interests to disclose.

\section{REFERENCES}

1. Miller SP, Ferriero DM, Leonard C, et al. Early brain injury in premature newborns detected with magnetic resonance imaging is associated with adverse early neurodevelopmental outcome. J Pediatr 2005;147:609-16.

2. Leviton A, Allred EN, Kuban KC, et al. Microbiologic and histologic characteristics of the extremely preterm infant's placenta predict white matter damage and later cerebral palsy. the ELGAN study. Pediatr Res 2010;67:95-101.

3. Shah DK, Doyle LW, Anderson PJ, et al. Adverse neurodevelopment in preterm infants with postnatal sepsis or necrotizing enterocolitis is mediated by white matter abnormalities on magnetic resonance imaging at term. J Pediatr 2008;153:170-5, 175.e1.

4. Glass HC, Bonifacio SL, Chau V, et al. Recurrent postnatal infections are associated with progressive white matter injury in premature infants. Pediatrics 2008;122:299-305.

5. Stoll BJ, Hansen NI, Adams-Chapman I, et al. National Institute of Child Health and Human Development Neonatal Research Network. Neurodevelopmental and growth impairment among extremely lowbirth-weight infants with neonatal infection. JAMA 2004;292:2357-65.

6. Adams E, Chau V, Poskitt KJ, et al. Tractography-based quantitation of corticospinal tract development in premature newborns. J Pediatr 2010;156:882-8, 888.e1.
7. Chau V, Poskitt KJ, McFadden DE, et al. Effect of chorioamnionitis on brain development and injury in premature newborns. Ann Neurol 2009;66:155-64.

8. Ellison VJ, Mocatta TJ, Winterbourn CC, et al. The relationship of CSF and plasma cytokine levels to cerebral white matter injury in the premature newborn. Pediatr Res 2005;57:282-86.

9. Back SA. Perinatal white matter injury: the changing spectrum of pathology and emerging insights into pathogenetic mechanisms. Ment Retard Dev Disabil Res Rev 2006;12:129-40.

10. Volpe JJ. Brain injury in premature infants: a complex amalgam of destructive and developmental disturbances. Lancet Neurol 2009;8:110-24.

11. Lehnardt S, Lachance C, Patrizi S, et al. The toll-like receptor TLR4 is necessary for lipopolysaccharide-induced oligodendrocyte injury in the CNS. J Neurosci 2002;22:2478-86.

12. Hoffmann O, Braun JS, Becker D, et al. TLR2 mediates neuroinflammation and neuronal damage. J Immunol 2007;178:6476-81.

13. Reiman M, Kujari H, Maunu J, et al.; PIPARI Study Group. Does placental inflammation relate to brain lesions and volume in preterm infants? J Pediatr 2008;152:642-7, 647.e1.

14. Lee SK, McMillan DD, Ohlsson A, et al. Variations in practice and outcomes in the Canadian NICU network: 1996-1997. Pediatrics 2000;106: 1070-9.

15. Soul JS, Hammer PE, Tsuji M, et al. Fluctuating pressure-passivity is common in the cerebral circulation of sick premature infants. Pediatr Res 2007;61:467-73.

16. Yanowitz TD, Potter DM, Bowen A, Baker RW, Roberts JM. Variability in cerebral oxygen delivery is reduced in premature neonates exposed to chorioamnionitis. Pediatr Res 2006;59:299-304.

17. Larouche A, Roy M, Kadhim H, et al. Neuronal injuries induced by perinatal hypoxic-ischemic insults are potentiated by prenatal exposure to lipopolysaccharide: animal model for perinatally acquired encephalopathy. Dev Neurosci 2005;27:134-42.

18. Patel S, Dammann O, Martin CR, Allred EN, Leviton A; ELGAN Study Investigators. Presumed and definite bacteremia in extremely low gestational age newborns. Acta Paediatr 2011;100:36-41.

19. Loron G, Olivier P, See H, et al. Ciprofloxacin prevents myelination delay in neonatal rats subjected to E. coli sepsis. Ann Neurol 2011;69:341-51.

20. Cornette LG, Tanner SF, Ramenghi LA, et al. Magnetic resonance imaging of the infant brain: anatomical characteristics and clinical significance of punctate lesions. Arch Dis Child Fetal Neonatal Ed 2002;86:F171-7.

21. Dyet LE, Kennea N, Counsell SJ, et al. Natural history of brain lesions in extremely preterm infants studied with serial magnetic resonance imaging from birth and neurodevelopmental assessment. Pediatrics 2006;118:536-48.

22. Stoll BJ, Hansen N, Fanaroff AA, et al. Late-onset sepsis in very low birth weight neonates: the experience of the NICHD Neonatal Research Network. Pediatrics 2002;110:285-91.

23. Papile LA, Burstein J, Burstein R, Koffler H. Incidence and evolution of subependymal and intraventricular hemorrhage: a study of infants with birth weights less than 1,500 gm. J Pediatr 1978;92:529-34.

24. Kreis R, Hofmann L, Kuhlmann B, et al. Brain metabolite composition during early human brain development as measured by quantitative in vivo 1H magnetic resonance spectroscopy. Magn Reson Med 2002;48:949-58.

25. Mukherjee P, Miller JH, Shimony JS, et al. Diffusion-tensor MR imaging of gray and white matter development during normal human brain maturation. AJNR Am J Neuroradiol 2002;23:1445-56.

26. Drobyshevsky A, Song SK, Gamkrelidze G, et al. Developmental changes in diffusion anisotropy coincide with immature oligodendrocyte progression and maturation of compound action potential. J Neurosci 2005;25:5988-97.

27. Team RDC 2007 R: A language and environment for statistical computing. R Foundation for Statistical Computing, Vienna.

28. 2010 R: Foundation for statistical computing. (http://www.R-Project.org.)

29. Burnham KP, Anderson DR. Understanding AIC and BIC in model selection. Sociol Methods Res 2004;33:261. 